\title{
Development of an Expert System to assist in Resource Management
}

\author{
Zenon Chaczko ${ }^{1}$, Lucia Carrion ${ }^{1}$, Wael Alenazy ${ }^{2}$, Mikayla $\mathrm{Mu}^{1}$ \\ ${ }^{1}$ Faculty of Engineering and Information Technology \\ University of technology, Sydney (UTS), Sydney, NSW, Australia \\ E-mail: \{Zenon.chaczko, Lucia.C.Carrion\}@uts.edu.au, Mikayla.Mu@alumni.uts.edu \\ ${ }^{2}$ Self-Development Skills Department, Preparatory Year Deanship \\ King Saud University (KSU) \\ Riyadh, The Kingdom of Saudi Arabia \\ E-mail: Walenazy@ksu.edu.sa
}

\begin{abstract}
This paper aims to demonstrate an idea of utilizing Kohonen Maps as a tool to portray and study resource allocations when constructing an expert system to assist in Resource Management. The context of work encompasses resource allocation and management tasks related to design of courses, as well as, various teaching and learning projects. The key aspect is to show the design of an expert system for resource allocation with the use of Kohonen Maps as an alternative way to visualise the demand and availability of project resources.
\end{abstract}

Keywords-Kohonen Maps; Self-Organizing Maps (SOM); Resource Allocation; Resource Demand

\section{INTRODUCTION}

In today's day and age of information technology it is important to various aspects of project management in academic environment. In this work, a particular focus is to show that a balanced management of project resources related to teaching and learning, as well as course planning activities can be achieved using a computer aided resource management tool(s) which can be made available to users at various levels. Inadequately calculated and allocated resources can cause serious delays in the academic (or student) project schedules and potentially affect project costs. Often, this happens due to having to source scarcely available resources or, at times, too many resources may have been allocated which can contribute to chaotic actions of project stakeholders.

The development of the Kohonen Maps based resource allocation system can enable a different approach of portraying the demand and availability of resources.

Kohonen Maps also known as Self-Organizing Maps (SOM) has a topological structure and is a computational model. Due to the Kohonen Maps topological structure it is called a topology-preserving map which is a map that preserves the neighbourhood relations [1] [2]. Based on the neural network Kohonen Maps have the ability to classify data without supervision in a self-organizing way [2]. Kohonen
Maps was considered as an idea to be used to display the usage of a resource including how popular that resource is based on how often it is demanded.

The use of Kohonen Maps will enable the user to view the demand for the resource by checking the density of the markers displayed in a generated graph. This graph will determine where the resource is required and used the most and which areas (time) they are more available (have more capacity).

Today project management software provides the user with real-time visibility into the project lifecycle [4].

Resource management is an essential component to ensure the success of a project. Different resource management strategies are used depending on the nature of the resource that is to be managed. Human resources possess certain skill sets required to do a job where as other resources such as equipment and materials are used to assist in completing a job. For resource management purposes, different resource types are considered [5]. When resources are effectively utilised, the goals of the project are achieved. The resources include the human resources and physical items including equipment and tools which are used to achieve the project objectives. Other resources include intangible items such as intellectual property, knowledge and skills. For any project the unavailability or lack of resources is a major obstacle.

This paper will examine the requirements for a resource allocation system, the designs for the resource allocation system portraying the resource demand, capacity and information. Also relates the development of a prototype of Kohonen Maps.

\section{PROBLEM DEFINITION}

\section{A. Methodology}

The method taken was based on the system lifecycle, on a prototype methodology and a research methodology for scoping.

The following steps were taken when completing this project: 
1. Planning - identifying objectives of this project, scope and requirements outlined, and deadlines defined.

2. Literature Review - main research component that considered how other Project Management systems function.

3. Designing the System - developed the conceptual design based on required functionality.

4. Implementation - coding the system.

5. Integration and Test - document, build and test the system.

6. Change Management - any changes were documented.

\section{B. Requirements for Resource Allocation System}

The following requirements for the resource allocation system were implemented:

- Use Kohonen Maps to display the resource allocation

- Display the availability of the resource over periods of time

- Display the tasks the resource is allocated to

- Have the option to display the resource allocation using graphs

- Display the resource information including allocations in a resource profile.

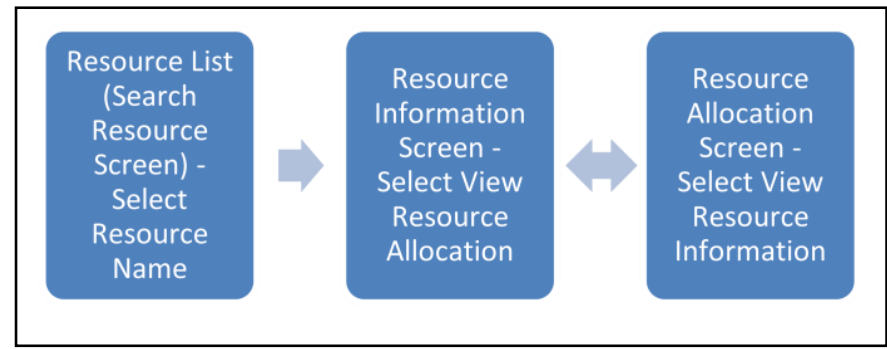

Fig. 1. Example of the Navigational Flow

\section{IMPLEMENTATION OF DESIGN}

Three screens were designed.

\section{A. Navigation}

In order to navigate to the Resource Information Screen, the user must select the resource's name (resource's name should be a hyperlink) from a Resource List (a search page) which would then load the Resource Information Screen. From the Resource Information Screen, the user can load the Resource Allocation Screen by clicking a link in this page.

\section{B. Search Resource Screen}

Searching for specific resources will be done via the Search Resource Screen. Fig. 2 shows an example of the Search Resource Screen where 'Test Person' will be used as the example resource.

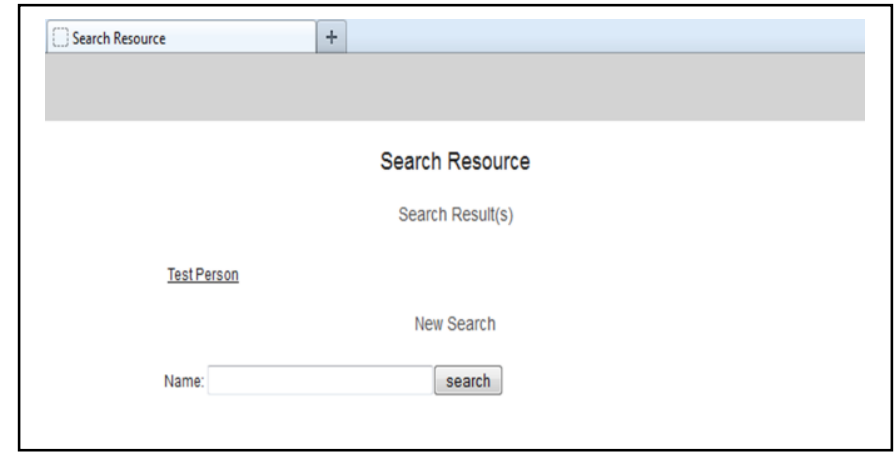

Fig. 2. Example of the Search Resource Screen

\section{Resource Information Screen}

Resource Information Screen should display the resource information ranging from their full name, their department, email, role, and location essentially it will act as a resource profile page. Fig. 3 conveys the resource information for 'Test Person'.

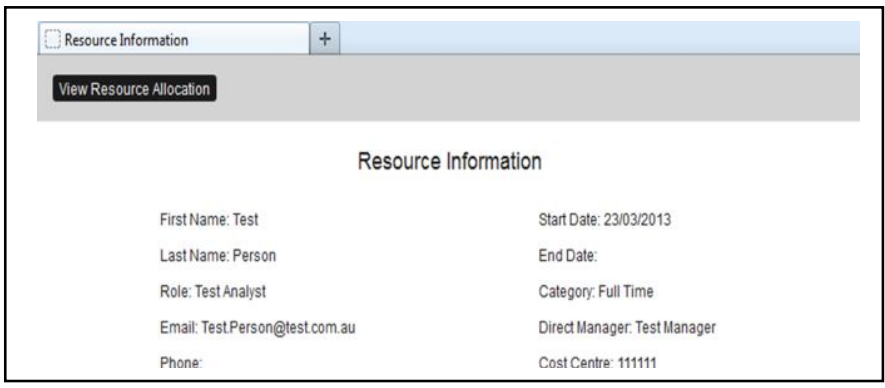

Fig. 3. Example of the Resource Information Screen

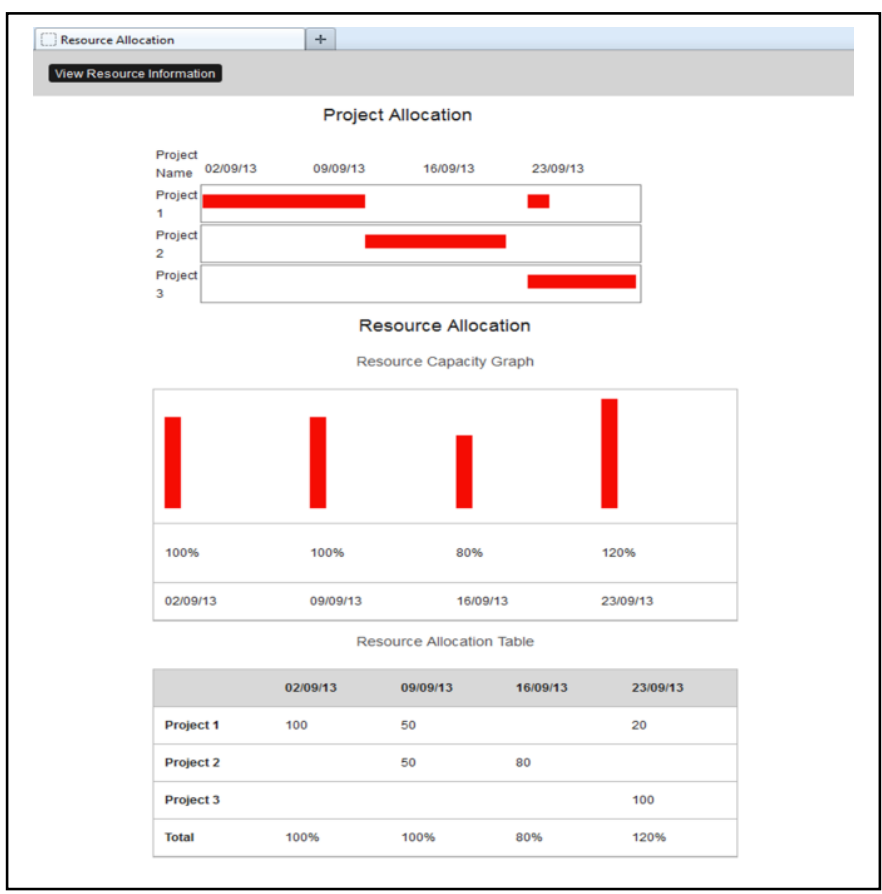

Fig. 4. Example of the Resource Allocation Screen 


\section{Resource Allocation Screen}

The Resource Allocation Screen should display the project allocation (bar graph), the resource capacity (column graph), and a table displaying the resource allocation (refer to Fig. 4).

The main idea will be incorporating Kohonen Maps and enabling it to display the resource allocations.

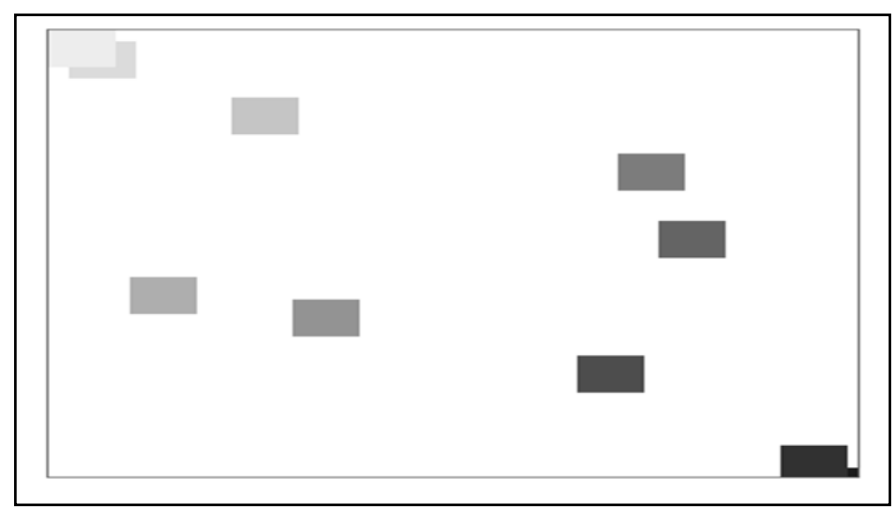

Fig. 5. Example of the Kohonen Map slightly modified from Adam Stirtan's work [3]

From Fig. 5 the Kohonen Map example portrays overlapping square shapes. This indicates a cluster and would convey an increase in resource demand, the greater the overlap the greater the resource demand.

The idea is to have the data for the graphs and the table be gathered from a database in order to generate the display.

The reason for designing a resource allocation system is so that the user can view a user friendly interface that is intuitive and will enable them to view the information they want at a glance. The basic idea is to create a system that is easy to use and relatively simple to implement.

The inclusion of the Kohonen Maps is different and could potentially show the demand of the system in a different way and could in future be used as a predictor of resource demand.

The use of Kohonen Maps could potentially path the way for a different and unique way of viewing resource demand as it can show a pictorial version of what the demand for the resource is. The basic design of the system would contain two main screens, the Resource Allocation Screen and the Resource Information Screen. Both screens will be connected to each other to enable the user to switch between the two screens.

\section{E. Resource Allocation in Projects}

When using project networks such as the program evaluation review technique (PERT) and the basic critical path method (CPM), both assume the availability of resources are unlimited.

When developing network schedules the resource requirements and the time constraints are considered. Since projects are restricted by three major limitations; resources, time, and performance requirements, trade-offs must be made as these limitations are challenging to satisfy concurrently. Poor resource allocation strategies can lead to quality of work being affected, and longer project schedules are expected with smaller resource bases.

As a guide, the use of the Pareto Principle (Pareto Law/Pareto Theory) can be used for planning, analysis, decision making and change management. The Pareto Principle is the phenomenon of an 80:20 rule discovered by Vilfredo Pareto (1848-1923) where the idea is that for example $20 \%$ of causes will produce a result of $80 \%$. In analysis the use of the Pareto Theory assists in prioritising possible changes. In the analysis you would identify the problems, the cause of the problems, give a score to each problem (the higher the score the higher the priority), group the problems together based on the cause (for example lack of staff is the cause for two problems then group them together), add the scores for each group, then take action to solve the problems. In regards to resource management this can assist in allocations, deciding which resources should be allocated to which tasks in order to produce the most benefit based on prioritisation. Since tradeoffs are made in projects, the Pareto method can assist in managing how best to utilise the resources, time, and performance requirements [6].

Using graphical representations to express information about resource assignment, utilisation and availability is part of resource profiling. Two common tools for resource profiling are resource levelling and resource loading graphs. Similarly the critical resource diagram and the resource idleness graph also express resource information.

\section{CODING IMPLEMENTATION}

\section{A. HTML, CSS and $C++$}

The Resource Allocation system was designed as a website with the idea in mind that users will access the resource allocation information via a network connected to a database.

HyperText Markup Language (HTML) and a Cascading Style Sheet (CSS) were used to design the website. Inspiration for the CSS was from Mack (2011). The CSS influences the design of the table, graph, general layout and presentation. The code for the website can be found in the appendix. Other sources including from TextFixer.com (2013) and Stanley (2007) provided useful information and inspiration regarding the HTML component.

There are examples of coding in different languages considering the behavior of the resources.

\section{B. HTML and CSS code}

\author{
/*General*/ \\ body \\ background: \#ffffff; \\ color: \#1e1e1e; \\ font: 12px/20px Arial, sans-serif; \\ margin: 0;
}


padding: 0 ;

h2

\{

font-size: 18px;

font-weight: normal;

line-height: 20px;

margin: 00 20px 0;

C. HTML-Search Resource Page (Index)

$<$ !doctype html>

$\langle$ html lang="en">

$<$ head>

$<$ meta charset="utf-8">

Page $\mid 43$

<meta name="viewport" content="width=1024">

$<$ title>Search Resource</title>

〈link rel="stylesheet" href="css.css">

$<$ /head >

$<$ body>

$<$ div id="wrapper">

$<\mathrm{p}>$

$<$ h2 $>$ Search Resource $</ h 2>$

$<$ table id="data-table" border="1" cellpadding="10" cellspacing="0"

summary="Search Results">

<caption>Search Result(s)</caption>

$</$ table $>$

<table style="width: 600px;">

$<\operatorname{tr}>$

$<$ td height="23" style="text-align: left; width: 100px;"><a

href="Resource Information.html" $>$ Test Person $</ a\rangle\langle/ \mathrm{td}\rangle$

<td style="text-align: left; width: 100px;">\&nbsp;</td >

$</$ tr $>$

$</$ table $>$

$\langle\mathrm{p}\rangle$

<table id="data-table" border="1" cellpadding=" 10 " cellspacing=" 0 "

summary="New Search">

<caption>New Search</caption>

$</$ table>

<form id="search box" form method="get" action="'>

Name:

\section{HTML - Information Page}

$<$ !doctype html >

$<$ html lang="en">
$<$ head $>$

$<$ meta charset="utf-8">

<meta name="viewport" content="width=1024">

$<$ title $>$ Resource Information $</$ title $>$

$<$ link rel="stylesheet" href="css.css">

$</$ head $>$

$<$ body>

$<$ div id="wrapper" >

$\langle\mathrm{p}\rangle$

$<$ h2 $>$ Resource Information $</ \mathrm{h} 2>$

<table style="width: 600px;">

$\langle\operatorname{tr}\rangle$

$<$ td height="23" style="text-align: left; width: 100px;">First Name: Test $</$ td $>$

\section{E. HTML-Resource Allocation Page}

$<$ !doctype html

$<$ html lang="en">

$<$ head $>$

<meta charset="utf-8">

<meta name="viewport" content="width=1024">

$<$ title $>$ Resource Allocation</title $>$

<link rel="stylesheet" href="css.css">

$</$ head $>$

<body>

<div id="wrapper">

<table style="width: 400px;">

$<\mathrm{tr}>$

$<$ td colspan="5">

$\langle$ div align $=$ "center" $>\langle$ h2 $>$ Project Allocation $\langle/$ h2 $\rangle\langle/$ div $\rangle$

$</$ td $>$

$</ \operatorname{tr}>$

$<\operatorname{tr}>$

<td style="width: 75px; vertical-align: bottom;">Project Name</td > <td style="width: 200px; vertical-align: bottom;">

<table style="width: 405px;">

$<\operatorname{tr}>$

<td height="23" style="text-align: left; width: 100px;">02/09/13</td> <td style="text-align: left; width: 100px;">09/09/13</td> <td style="text-align: left; width: 100px;">16/09/13</td> <td style="text-align: left; width: 100px;">23/09/13</td >

$</ \operatorname{tr}>$

$</$ table $>$

$</$ td $>$

$</ \operatorname{tr}>$ 


\section{F. $C++-$ Kohonen Maps}

The following code has been slightly modified from Adam Stirtan's (2010) work in orderincorporate a new item in the dropdown menu. In order to initialise the images for the Kohonen Maps the following code was used to initialise the settings to create a new image list and directory:

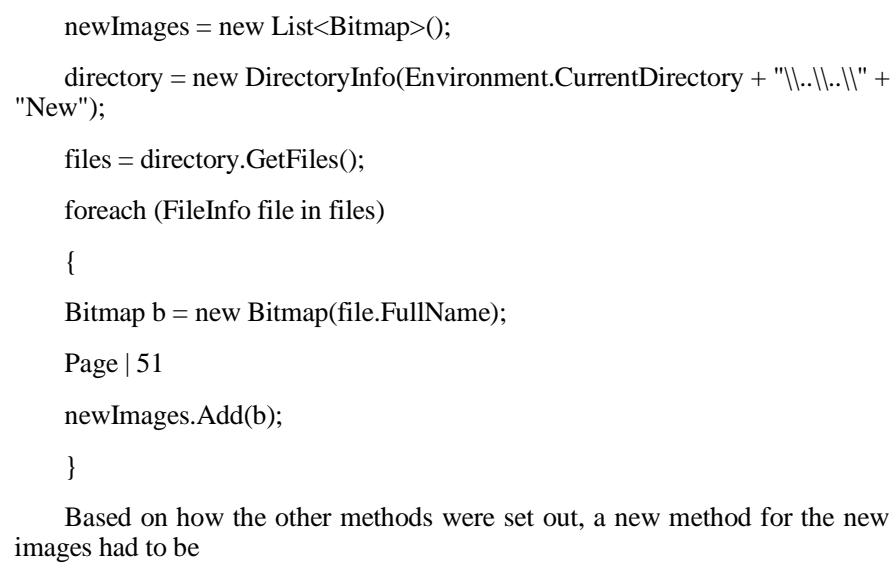

Starting from zero, there is a total of three if statement options for this particular menu dropdown with newImages being the last option (option two). Hence for newImages to have been selected the third option must be selected. The images have been scaled by 3 in order to reduce the size of the image when displayed in the application (images are originally 100px by 100px).

\section{FUTURE DEVELOPMENT}

Future improvements for Kohonen Maps could include:

- Integration of the executable file into the web page as an embedded interactive file

- Utilised as a predictor of future resource demand by basing it on past trends

- Potential expansion or integration into a project management system

- Analyse the resource demand for projects based on a particular period

- Analyse the web traffic each page in the system receives.

- Enable adaptability to other systems. This idea can be improved to analyse the resource and how often a page in the system is viewed.

\section{CONCLUSIONS}

The development of a web based resource allocation system is something that will be useful for projects in order to manage a resource's capacity. The utilisation of Kohonen Maps will be different and is something that could assist projects in the future.

Labour resources need to be effectively managed in order to be utilised efficiently minimise redundant costs in training design and development of a resource allocation system assists users in managing, controlling, and allocating resources.

Resource allocation is a difficult task, tool through the verification and validation of existing project management tools create a web based system utilising Kohonen Maps as a unique feature to enable a different way of viewing the resource demand.

Important measurements are applied to all projects by corporations that perform exceedingly well in project delivery. These measurements include return on investment, creating value and accomplishing business benefits.

The complexity of resource management is constrained by limitations such as resource interdependencies, limitations on resource availability and substitutions, resource priorities and mutual exclusivity of resources.

The constraints determine the techniques and tools that are used for resource management. If the human resource does not have the proper equipment and other resources, the work won't be completed. 


\section{REFERENCES}

[1] Orr, G., Schraudolph, N. \& Cummins, F. 2013, Kohonen's SelfOrganizing Map (SOM), Willamette University, Oregon, viewed 5 May 2013 ,

<http://www.willamette.edu/ gorr/classes/cs449/Unsupervised/SOM.h $\mathrm{ml}>$.

[2] Rojas, R. 1996, Neural Networks, Freie Universität Berlin, Berlin, viewed $\quad 5 \quad$ May $2013 \quad<$ http://page.mi.fuberlin.de/rojas/neural/chapter/K15.pdf>.

[3] Adam Stirtan 2010, Kohonen Network Self Organizing Map for Image Clustering, video recording, viewed 18 November 2013 , <http://www.youtube.com/watch?v=2YTco1z_BGU>.
[4] Hewlett-Packard Development Company, L.P. 2010, HP Project and Portfolio Management Center, December 2010, cat. no. 4AA09340ENW, Hewlett-Packard Development Company, L.P., Palo Alto, viewed $17 \quad$ March 2013, <http://www.ts.avnet.com/uk/vendors/hp/assets/hp_saas_project_portfol io_management_centre_brochure.pdf $>$.

[5] Badiru, A. B., Badiru, A. \& Badiru, A. 2008, Industrial Project Management: Concepts, Tools, and Techniques, Taylor \& Francis Group, Boca Raton.

[6] Mind Tools Ltd 2013, Pareto Analysis, viewed 16 June 2013, <http://www.mindtools.com/pages/article/newTED_01.htm>. 